\title{
Testing of almost all the hadronic interaction models by comparing calculated muon energy spectrum with data
}

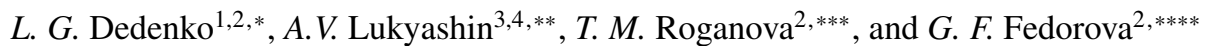 \\ ${ }^{1}$ Faculty of Physics M.V. Lomonosov Moscow State University, Leninskie Gory, 119991 Moscow, Russia \\ ${ }^{2}$ Skobeltsyn Institute of Nuclear Physics, Lomonosov Moscow State University, 119234 Moscow, Russia \\ ${ }^{3}$ Institute for Theoretical and Experimental Physics named by A.I. Alikhanov of National Research Center "Kurchatov Institute", \\ 117218 Moscow, Russia \\ ${ }^{4}$ National Research Nuclear University (MEPhI) Moscow Engineering Physics Institute, 115409 Moscow, Russia
}

\begin{abstract}
Uncertainties of the model energy spectra of the most energetic secondary charged mesons are discussed. Computer simulations of the partial energy spectra of the atmospheric vertical muons induced by primary cosmic particles with various fixed energies in terms of hadronic interactions models had been carried out with the help of the CORSIKA package. These partial spectra have been convolved with the contemporary spectra of the primary cosmic particles in the energy range 0.1-10 $000 \mathrm{TeV}$. Results of simulations are compared with the contemporary data of the atmospheric vertical muon flux. Comparison shows that all models underestimate the production of secondary charged $\pi^{ \pm}$-mesons (and $K^{ \pm}$-mesons) by a factor of $\sim 1.4 \div 2$ at the highest energies. This underestimation induces a more rapid development of extensive air showers in the atmosphere and results in uncertainties in estimates of energy and composition of the primary cosmic particles.
\end{abstract}

\section{Introduction}

Extensive air showers (EAS) are the only tool to understand the origin and composition of cosmic rays, their possible sources and the transport of cosmic particles in various magnetic fields on their way to the Earth at very high energies. All features of the energy spectrum, arrival directions and composition of the primary cosmic particles should be determined through an analysis of the EAS data. These data as signals in the surface and underground detectors are usually interpreted in terms of various models of hadronic interactions [1-9]. It happened, that such interpretation leads to some inconsistency. As an example, the energy of showers calculated in terms of the QGSJET II-03 [3] model with the help of the surface detectors signals at the Telescope Array [10] happened to be 1.27 times lager than this energy estimated with help of the fluorescence light. To ensure that results of such interpretation are as accurate as possible these models should be thoroughly tested. Usually these models are tested with the help of accelerator data at small values $(\sim 0)$ of the pseudorapidity, $\eta$, where most of secondary particles (mainly mesons) are produced [11-13]. However, calculations have shown that the maximal energy flow carried by secondary particles occurs at much larger values $(\sim 8-10)$ of the pseudorapidity $\eta$ [14]. Let us also note that the longitudinal development of EAS de-

\footnotetext{
*e-mail: ddn@dec1.sinp.msu.ru

**e-mail: lukyashin.anton@physics.msu.ru

***e-mail: ragatm@yandex.ru

****e-mail: fdr@dec1.sinp.msu.ru
}

pends strongly on the rate of the projectile particle energy fragmentation. The atmospheric muon flux also depends strongly on this production of the highest energy mesons. So, it is of primary importance to verify a production of the most energetic mesons simulated in terms of various models. This verification may be carried out by comparing model predictions of these muon fluxes with data. We select the classical experiments L3+Cosmic [15], MACRO [16], LVD [17] and IceCube [18] and elaborate the smooth approximation of these muon data in the energy interval of $10^{2}-10^{5} \mathrm{GeV}$. The model predictions of muon flux have been estimated as follows. Showers induced by primary protons and helium nuclei with different fixed energies have been simulated with the help of the CORSIKA package [19] and the muon partial energy spectrum in each individual shower have been calculated. Results of these simulations for every type of primary particles multiplied by intensities of these particles should be integrated on the energy of particles. Thus, we also need some expressions for the energy spectra of various primary particles. Inspired by modern results and a new precise cosmic ray data base [20], we suggested new approximations of cosmic ray energy spectra for primary protons and helium nuclei. Indeed, there are results of many measurements of the fluxes of the primary cosmic nuclei (e.g.AMS-02 [21], PAMELA [22], ATIC2 [23], CREAM [24], ARGO-YBJ [25], ARGO-YBJ \& FWCTA [26], KASCADE [27], KASCADE-Grande [28], Tunka [29], IceCube [30], Telescope Array [31]). Besides there are some calculations of spectra of the primary proton and helium nuclei in SNR [32]. We will use these ap- 


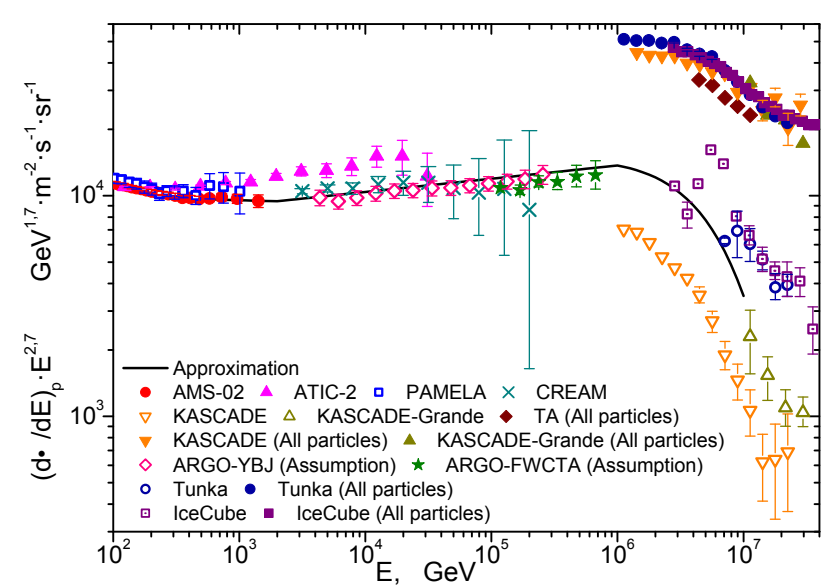

Figure 1. Energy spectra of the primary protons. Solid line suggested approximation. All particle spectra depends on the energy per particle.

proximations for the energy spectra of the primary protons and helium nuclei to estimate convolutions with the partial muon spectra. Thus, with the help of any of the interaction models [1-9], the CORSIKA package and approximations of data on fluxes of the primary cosmic nuclei [21-31] one can predict the energy spectra of atmospheric vertical high energy muons at sea level. These predictions can be compared with data observed by the L3+Cosmic, MACRO and LVD collaborations at energies above $100 \mathrm{GeV}$. Finally, some conclusion can be drawn about the validity of various models.

In fact, some low energy models with the FLUKA package [33] have been tested in such a way. We are sorry that some our results of models testing in [34-36] are not correct. We do apologize for our mistake in input data for the atmosphere.

In this paper the eight models: QGSJET01 [1], QGSJET II-03, QGSJET II-04 [4], DPMJET 2.55 [5], VENUS 4.12 [6], EPOS LHC [7, 8], SIBYLL 2.1 and SIBYLL 2.3 [9] have been tested. A comparison of muon data observed in [15-18] with results of simulations allows to draw a conclusion about the most energetic meson production described by these models.

\section{Method}

To estimate the energy spectra $D\left(E_{\mu}\right)$ of atmospheric vertical muons in the energy range of $10^{2}-10^{5} \mathrm{GeV}$ we need to know the energy spectra $d I_{p} / d E$ and $d I_{H e} / d E$ of the primary protons and helium nuclei within the energy interval $10^{2}-10^{7} \mathrm{GeV}$ and the partial energy spectra $S_{p}\left(E_{\mu}, E\right)$ and $S_{H e}\left(E_{\mu}, E\right)$ of the vertical muons in EAS induced by the primary protons and helium nuclei with various fixed energies, $E$. Simulations of these partial muon spectra have been carried out in terms of the QGSJET01, QGSJET II-03, QGSJET II-04, DPMJET 2.55, VENUS 4.12, EPOS LHC, SIBYLL 2.1 and SIBYLL 2.3 hadronic interaction models in the same energy range of $10^{2}-10^{7} \mathrm{GeV}$. The smooth approximation of the atmospheric muon data observed by the L3+Cosmic,

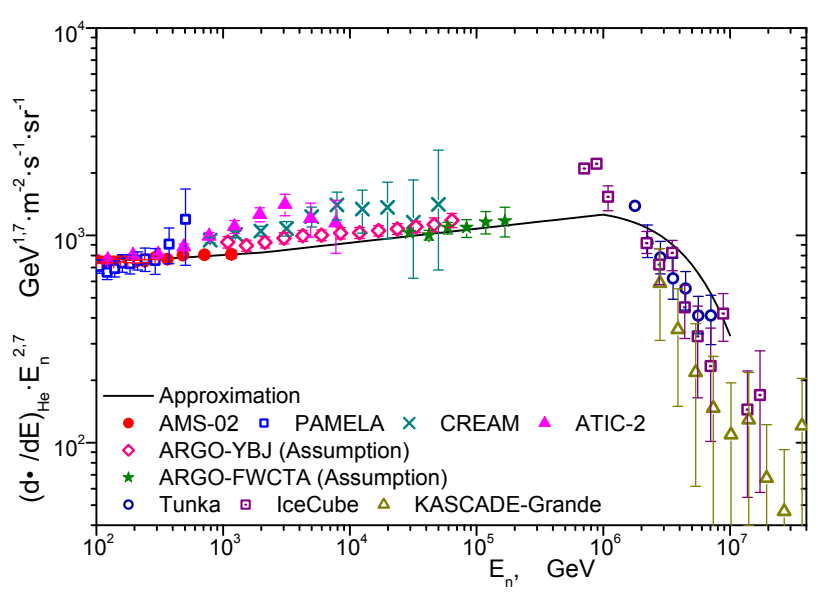

Figure 2. Energy spectra of the primary helium nuclei. Solid line - suggested approximation.

Table 1. Parameters of approximation for the primary nucleon spectra.

\begin{tabular}{lllllll}
\hline Nuclei & $K_{1}$ & $K_{2}$ & $R_{1}$ & $R_{2}$ & $\alpha$ & $s$ \\
\hline $\mathrm{H}$ & 0.4544 & 5833 & 45 & 336 & 2.849 & 2.64 \\
$\mathrm{He}$ & 0.1896 & 493 & 22.5 & 122.5 & 2.78 & 2.623 \\
\hline
\end{tabular}

MACRO and LVD collaborations have been used for comparison with the results of simulations.

Functions $S_{p}\left(E_{\mu}, E\right)$ and $S_{H e}\left(E_{\mu}, E\right)$ are the partial differential energy spectra of muons in showers induced by primary protons and helium nuclei with fixed values of energy $E$. These spectra were calculated for 24 different fixed values of energy $E$ of the primary protons. The energy distributions of muons induced by primary helium nuclei $S_{H e}\left(E_{\mu}, E\right)$ have also been calculated and compared with simulations based on the hypothesis of superposition [37]. As direct results coincide with simulations in terms of the hypothesis of superposition, we will use this hypothesis.

The energy spectra of the primary particles are important ingredients of simulations. As the energy per nucleon is of importance only the energy spectra of the primary protons and helium nuclei should be taken into account. We have used approximations (eqn. (1)) for $\left(d I_{p} / d E\right)$ and $\left(d I_{H e} / d E\right)$ based on Berezhko calculations [32] and normalized at AMS-02 data at 1.8 TeV [21]. Figures 1 and 2 demonstrate how these approximations fit the data [21$31]$.

To take into account a possible change of primary spectrum above the "knee" at energies above $E_{1} \simeq 10^{6}$ $\mathrm{GeV}$ for the primary protons and helium nuclei we have used an additional exponential multiplier. The values of parameters for these new approximations are listed in Table 1 . For primary protons $\beta=5.5417$ and $\gamma=0.024$. For primary helium nuclei $\beta=4.4074$ and $\gamma=0.027$.

The approximation parameters: $R_{1}, R_{2}, \alpha, s, \beta$ and $\gamma$ are dimensionless. Parameters $K_{1}$ and $K_{2}$ are dimensional as $\left[1 /\left(G e V \cdot m^{2} \cdot s \cdot s r\right)\right]$. The notation $E$ is the kinetic energy per nucleon in $\mathrm{GeV}$. 


$$
\frac{d I}{d E}= \begin{cases}K_{1} \cdot\left(\frac{E}{R_{1}}\right)^{-\alpha} \cdot\left[1+\left(\frac{E}{R_{2}}\right)^{\beta}\right]^{\gamma} & E \in 10^{2} \div 1.8 \cdot 10^{3} \mathrm{GeV} \\ K_{2} \cdot E^{-s} & E \in 1.8 \cdot 10^{3} \div 10^{6} \mathrm{GeV} \\ K_{2} \cdot E^{-s} \cdot \exp \left[\frac{-\left(E-10^{6}\right)}{6 \cdot 10^{6}}\right] & E \in 10^{6} \div 10^{7} \mathrm{GeV}\end{cases}
$$

The CORSIKA 7.4 package (CORSIKA 6.9 in the case of DPMJET 2.55 and QGSJET II-03 models and CORSIKA 7.56 in the case of SIBYLL 2.3 model) have been used to simulate the second important ingredients - the partial energy spectra $S_{p}\left(E_{\mu}, E\right)$ and $S_{H e}\left(E_{\mu}, E\right)$ of vertical muons in showers induced by the primary protons and helium nuclei with various fixed energies, $E$, in terms of the eight models in the energy range $10^{2}-10^{5} \mathrm{GeV}$ with statistics $10^{6}$ events for the most energetic muons.

The results of these calculations in the energy range $10^{2}-10^{7} \mathrm{GeV}$ were interpolated for 100 values of energies $E$ with equal intervals in decimal logarithmic scale. The energy interval $10^{2}-10^{5} \mathrm{GeV}$ of muons was divided into 60 equal bins also in decimal logarithmic scale. So, the width of the bin was equal to $h=\lg \left(E_{\mu,(i+1)} / E_{\mu, i}\right)=0.05$. Let us note that average muon energies for the $1^{s t}, 21^{s t}$ and $41^{\text {st }}$ bins we will use later are equal to $1.059 \cdot 10^{2}, 1.059$. $10^{3}$ and $1.059 \cdot 10^{4} \mathrm{GeV}$ respectively. In fact simulations for helium nuclei have been carried out only for energies $10^{4}$ and $10^{6} \mathrm{GeV}$ to test the hypothesis of superposition. As results of simulations for the primary nuclei showed a good agreement with this hypothesis we have used this hypothesis to estimate the flux of the nucleons from the primary helium nuclei.

The energy spectra $D_{p}\left(E_{\mu}\right)$ and $D_{H e}\left(E_{\mu}\right)$ of muons for primary protons and helium nuclei are calculated as integrals of products of functions $S_{p}\left(E_{\mu}, E\right)$ and $S_{H e}\left(E_{\mu}, E\right)$ with corresponding intensities $d I_{p} / d E$ and $d I_{H e} / d E$ of the primary protons, on energy $E$ of primary nucleons.

$$
\begin{gathered}
D_{p}\left(E_{\mu}\right)=\int\left(\frac{d I_{p}}{d E}\right) \cdot S_{p}\left(E_{\mu}, E\right) \cdot d E \\
D_{H e}\left(E_{\mu}\right)=\int\left(\frac{d I_{H e}}{d E}\right) \cdot S_{H e}\left(E_{\mu}, E\right) \cdot d E
\end{gathered}
$$

The resulting energy spectrum of atmospheric muons is the sum of these energy spectra of muons produced by primary protons and helium nuclei.

$$
D\left(E_{\mu}\right)=D_{p}\left(E_{\mu}\right)+D_{H e}\left(E_{\mu}\right)
$$

\section{Results of simulations}

The partial energy spectra $S_{p}\left(E_{\mu}, E\right)$ of the atmospheric vertical muons simulated for various fixed energies $E$ of the primary protons in terms of the EPOS LHC model are shown in figure 3 . It is seen that statistics of $\sim 10^{6}$ at the higher energy end (tail) of the spectra is not enough.

Table 2 displays the total number of muons with energies above $10^{2}$ and $10^{3} \mathrm{GeV}$ in showers induced by primary protons with energies $10^{5}$ and $10^{6} \mathrm{GeV}$ estimated in

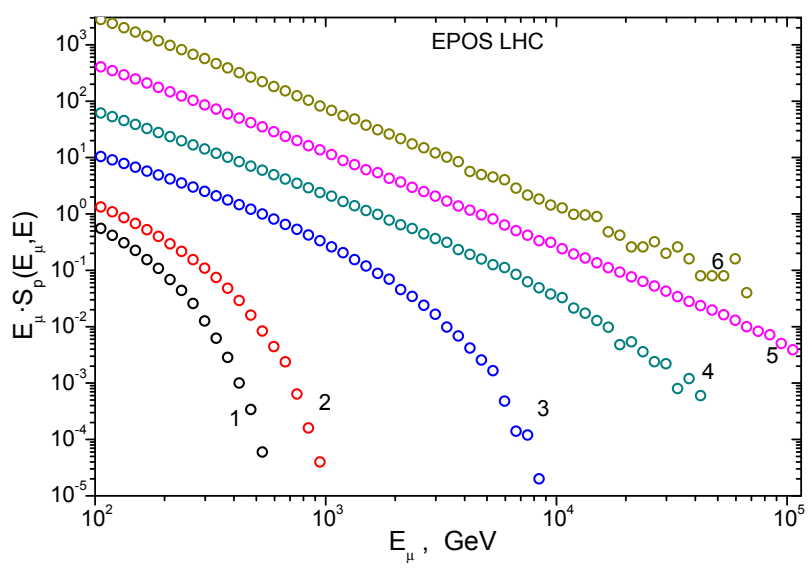

Figure 3. The partial energy spectra of muons in showers induced by primary protons with various fixed energies E: $1-5 \cdot 10^{2}$ $\mathrm{GeV} ; 2$ - $10^{3} \mathrm{GeV} ; 3-10^{4} \mathrm{GeV} ; 4-10^{5} \mathrm{GeV} ; 5$ - $10^{6} \mathrm{GeV} ; 6$ $10^{7} \mathrm{GeV}$.

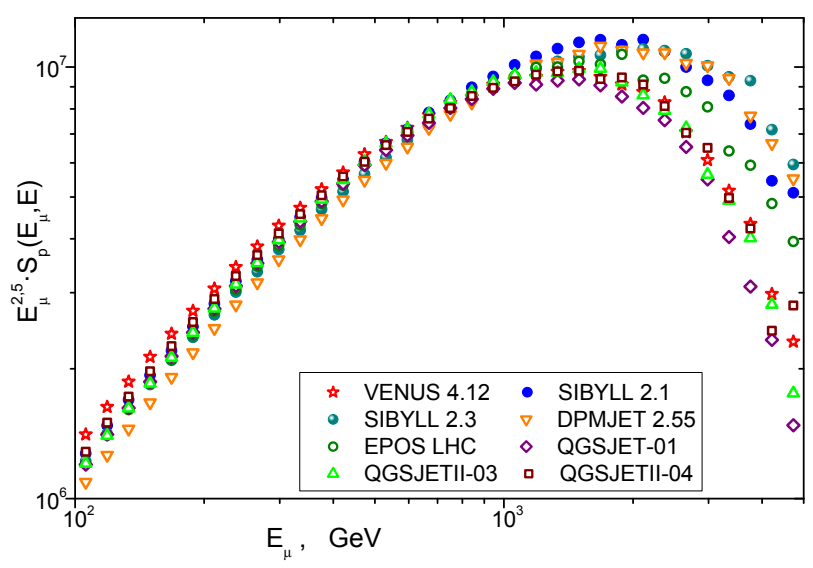

Figure 4. The partial energy spectra of muons in showers induced by primary protons with fixed energy $\mathrm{E}=10^{4} \mathrm{GeV}$.

terms of the eight models in our simulations and in [38]. A very reasonable agreement is seen.

The next figure 4 demonstrates a comparison of the partial muon energy spectra $S_{p}\left(E_{\mu}, E\right)$ calculated in terms of the eight models for a fixed energy $E=10^{4} \mathrm{GeV}$ of the primary protons. The results for the SIBYLL 2.1 model are the highest, and the QGSJET01 model values are the lowest at muon energy $E_{\mu} \simeq 10^{3} \mathrm{GeV}$. The result of the rest of models are in between these two models.

It is important to estimate energy intervals of the primary protons which contribute into various bins of the muon energy spectrum. But first some dependence of muon numbers inside the partial bins on energy $E$ should be illustrated.

Figure 5 demonstrates the distributions of the primary proton energy $E$ for the bins of muon energy spectra for the SIBYLL 2.3 model. Let us remember that average energies of muons for these bins are equal to $1.059 \cdot 10^{2}$, $1.059 \cdot 10^{3}$ and $1.059 \cdot 10^{4} \mathrm{GeV}$ accordingly. It is possible to note that nearly two orders of energy $E$ are of importance 
Table 2. Average number of muons with energies above the threshold $E_{t h}$ in showers induced by primary protons with energies $E$.

\begin{tabular}{lllllll}
\hline & & \multicolumn{2}{|c|}{$E=10^{5} \mathrm{GeV}$} & \multicolumn{2}{c}{$E=10^{6} \mathrm{GeV}$} \\
\hline Model & Paper & $E_{\text {th }}$ & $100 \mathrm{GeV}$ & $1000 \mathrm{GeV}$ & $100 \mathrm{GeV}$ & $1000 \mathrm{GeV}$ \\
\hline VENUS 4.12 & {$[38]$} & & 23,5 & 0,679 & 153,5 & 3,932 \\
VENUS 4.12 & This work & 24,5 & 0,652 & 156,2 & 3,839 \\
QGSJET01 & {$[38]$} & 21,0 & 0,605 & 132,3 & 3,612 \\
QGSJET01 & This work & 20,9 & 0,593 & 132,8 & 3,490 \\
QGSJET II-03 & This work & 20,3 & 0,592 & 122,8 & 3,301 \\
QGSJET II-04 & This work & 22,4 & 0,613 & 141,3 & 3,584 \\
DPMJET 2.55 & This work & 18,7 & 0,538 & 116,7 & 3,150 \\
SIBYLL 2.1 & This work & 21,6 & 0,6 & 131,4 & 3,515 \\
SIBYLL 2.3 & This work & 21,4 & 0,573 & 136,2 & 3,461 \\
EPOS LHC & This work & 20,3 & 0,576 & 128,2 & 3,206 \\
\hline
\end{tabular}

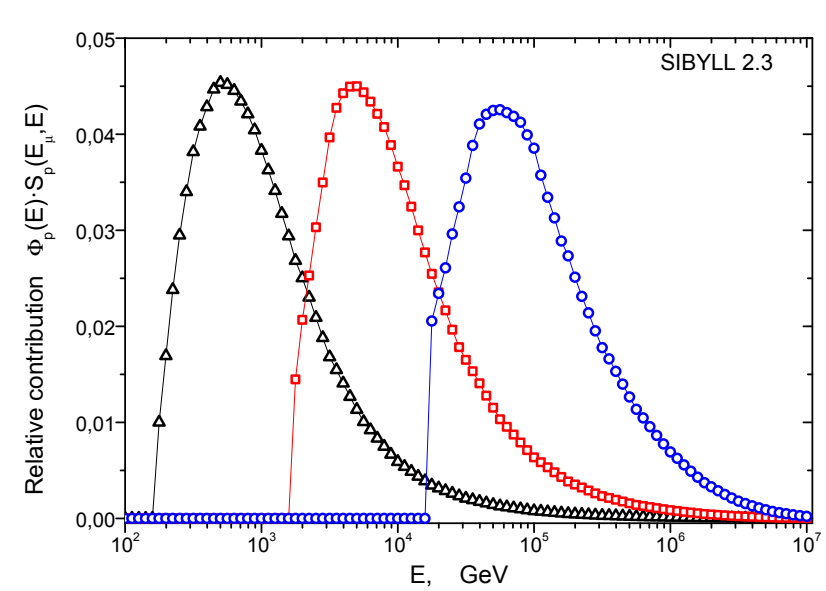

Figure 5. The energy distributions of primary protons contributed to three bins with muon average energies: $\triangle-1.059 \cdot 10^{2}$ $\mathrm{GeV}, \square-1.059 \cdot 10^{3} \mathrm{GeV}$ and $\circ-1.059 \cdot 10^{4} \mathrm{GeV}$.

for any fixed muon energy $E_{\mu}$. The maximal contributions occur at energies $\sim 5 \cdot 10^{2} \mathrm{GeV}$ (to the $1^{\text {st }}$ bin), $\sim 5 \cdot 10^{3}$ $\mathrm{GeV}$ (to the $21^{s t}$ bin) and $\sim 5 \cdot 10^{4} \mathrm{GeV}$ (to the $41^{s t}$ bin).

The final results of the muon energy spectra $D\left(E_{\mu}\right)$ calculated in terms of the eight hadronic interaction models and data [15-18] are shown in figure 6. The ratios of MC simulation to data are shown in figure 7 .

It should be noted that at energies above $\sim 100 \mathrm{GeV}$ both the simulated spectra and data are steepened. It is because the decay constant $B$ for the charged mesons is equal to $B \sim 100 \mathrm{GeV}$ and the probability of decay for charged mesons is decreasing at higher energies.

It should be noted that contributions to the generation of muons into the $21^{s t}$ bin with an average energy of $E_{\mu} \simeq 10^{3} \mathrm{GeV}$ are most considerable from primary protons with energy $E=10^{4} \mathrm{GeV}$ as shown in figure 5. So, various models may be effectively compared at energies at which their contributions into the muon spectrum is most considerable. Such comparison of relative contributions of the eight models to the generation of muons into the $21^{s t}$ bin are shown in figure 4 and corresponds to the final result in the same energy region shown in figure 7.

It is seen that calculated spectra are $\sim 2$ times below data in the case of the QGSJET01 model and $\sim 1.4$

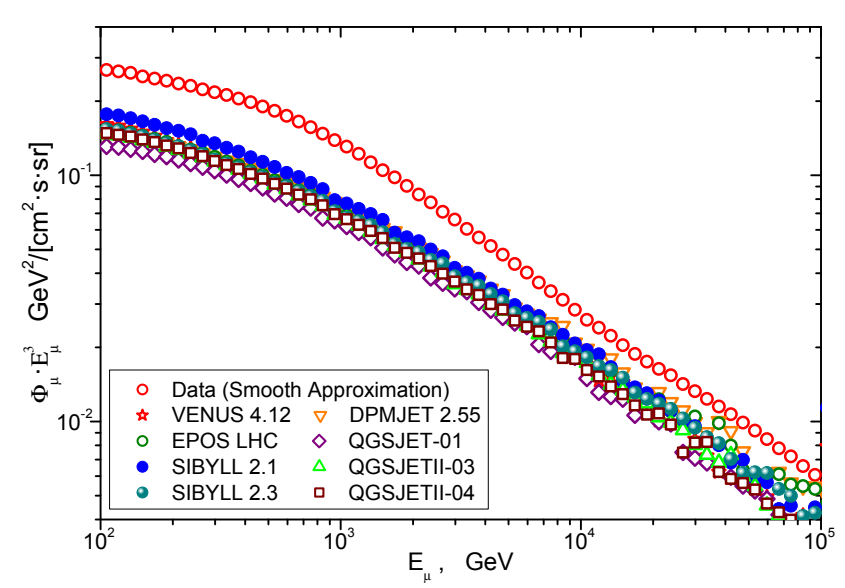

Figure 6. The energy spectra of near vertical muons.

times below data for the SIBYLL 2.1 model. The results of DPMJET 2.55 model is $\sim 1.86$ below data at energies $E_{\mu} \simeq 10^{2} \mathrm{GeV}$ and $\sim 1.36$ below data at energies $E_{\mu} \simeq 10^{4}$ $\mathrm{GeV}$. The results of QGSJET01 model is $\sim 2$ below data at energies $E_{\mu} \simeq 10^{2} \mathrm{GeV}$ and $\sim 1.7$ below data at energies $E_{\mu} \simeq 10^{4} \mathrm{GeV}$. The results of SIBYLL 2.1 model is $\sim 1.5$ below data at energies $E_{\mu} \simeq 10^{2} \mathrm{GeV}$ and $\sim 1.35$ below data at energies $E_{\mu} \simeq 10^{4} \mathrm{GeV}$. The result of the rest of models are in between these limits and slightly different from each other by $\sim 10-15 \%$. The main conclusion is quite clear. All considered models demonstrate the valuable deficit of muons [39-43].

In this paper the muon fluxes intensities for the DPMJET 2.55, QGSJET II-03, SIBYLL 2.3 and VENUS 4.12 models differ by $\sim 2 \div 15 \%$ with an increase in energy from $\sim 10^{2} \mathrm{GeV}$ to $\sim 10^{4} \mathrm{GeV}$ compared with the work of Ref. [42, 43] due to slightly different modified approximations of the primary cosmic ray spectrum.

\section{Conclusion}

Muons which contribute much to the muon energy spectra are produced in decays of the most energetic $\pi^{ \pm}$-mesons and $K^{ \pm}$-mesons generated in first interactions of the primary particles with nuclei in the atmosphere. As calculated vertical muon energy spectra in the case of the 


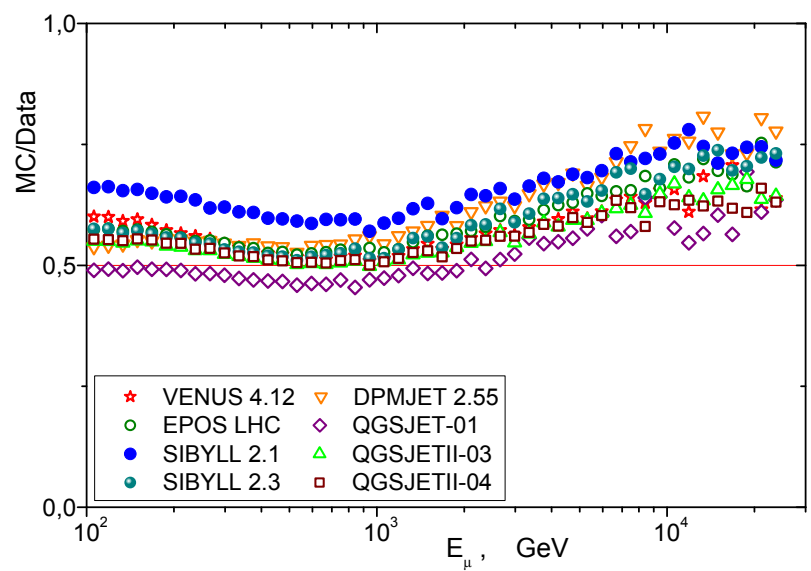

Figure 7. The ratio MC/DATA.

eight (QGSJET01, QGSJET II-03, QGSJET II-04, DPMJET 2.55, VENUS 4.12, EPOS LHC, SIBYLL 2.1 and SIBYLL 2.3) hadronic interaction models are $\sim 1.4 \div 2$ times below data we can conclude that production of the most energetic $\pi^{ \pm}$-mesons and $K^{ \pm}$-mesons in these models is considerably suppressed. This suppression may induce smaller values of signals in the surface scintillation detectors and will result in larger values of the calculated energy estimates. So, the coefficient 1.27 used by the TA collaboration [10] to decrease the energy estimates of showers calculated on the basis of signals in the surface detectors may be understood as a result of this suppression. The increased intensity of the primary particle flux observed at the Yakutsk array at super high energies [44] may also be a result of smaller values of calculated signals in surface scintillation detectors.

\section{Acknowledgement}

The authors thank N. N. Kalmykov who kindly showed alternative calculations and A. A. Lagutin for important assistance in the verification of the results for the QGSJET01 model.

\section{References}

[1] Kalmikov N. N., Ostapchenko S. S., Phys. Atom. Nucl. Vol. 56, 346 (1993).

[2] Ostapchenko S. S., Nucl. Phys. B (Proc. Suppl.) Vol. 151, 143 (2006).

[3] Ostapchenko S. S., Phys. Rev. D. Vol. 74, 014026 ; hep-ph/0505259 (2006).

[4] Ostapchenko S. S., Phys. Rev. D. Vol. 83, 014018 (2011).

[5] Ranft J., Phys. Rev. D. Vol. 51, 64 (1995); hepph/9911213 (1999).

[6] Werner K., Phys. Rep. Vol. 232, 87 (1993).

[7] Werner K., Nucl. Phys. B (Proc. Suppl.) Vol. 175, 81 (2008).

[8] Werner K. Liu F. M. and Pierog T., Phys. Rev. C. Vol. 74, 044902; hep-ph/0506232(2006).
[9] Ahn E.-J., Engel R., Gaisser T. K., et al., Phys. Rev. D. Vol. 80, 094003 (2009).

[10] Abu-Zayyad T., Aida R., Allen M., et.al., (Telescope Array Collab.) Astrophys. J. Lett., Vol. 768:L1, 5 (2013).

[11] Pierog T., EPJ Web of Conf., Vol. 99, 09002 (2015).

[12] Ostaphcenko S. S., Progr. of Theor. Phys. Suppl., Vol. 193, 204 (2012).

[13] D’Enterria D., Engel R., Pierog T., et. al., Astropart. Phys., Vol. 35, 98 (2011).

[14] Engel R., Rebel H., Acta Phys. Polonica B., Vol. 35, 321 (2004).

[15] Achard P. Adriani O., Aguilar-Benitez M., et al.,(L3 Collab.), Phys.Lett. B. 598, 15-32; hep-ex/0408114v1K (2004).

[16] Ambrosio M., Antolini R., Auriemma G., et al., (MACRO Collab.), Phys. Rev. D. Vol. 52, 3793 (1995).

[17] Aglietta M., Alpat B., Alieva E. D., et al., (LVD Collab.), Phys.Rev. D. 58, 092005; hep-ex/9806001v1 (1998).

[18] Tenholt F., et al., (Ice Cube Collab.) Proceedings of the 35th International Cosmic Ray Conference, (Busan, Korea 2017) PoS(ICRC2017) 317.

[19] Heck D., Knapp J., Capdevielle J.-N., et al., Report FZKA 6019 Forschungszentrum Karlsruhe, (1998).

[20] Maurin D., Melot F. and Taillet R., Astronomy and Astrophysics, 569, A32 (2014).

[21] Aguilar M., Aisa D., Alpat B., et al, (AMS Collab.) Phys. Rev. Lett. Vol. 114, 171103 (2015). Aguilar M., Aisa D., Alpat B., et al, (AMS Collab.) Phys. Rev. Lett. Vol. 115, 211101 (2015).

[22] Adriani O., Barbarino G. C., Bazilevskaya G. A., et. al., (PAMELA Collab.) Advances in Space Research Vol. 51, 219-226 (2013).

[23] Panov A. D., Adams J. H., Ahn H. S. et al. (ATIC Collab.), Bull. Russ. Acad. Sci. Phys. Vol. 71, 494 (2007); Bull. Russ. Acad. Sci. Phys. Vol. 73, 564 (2009).

[24] Ahn H. S. Allison P., Bagliesi M. G., et al (CREAM Collab.), , Astrophys.J., 707, 593 (2009).

[25] Bartoli B., Bernardini P., Bi X., et al (ARGOYBJ Collab.) Phys. Rev. D 91, 11, 112017; arXiv:1503.07136 (2015). Di Sciascio G. et. al. (ARGO-YBJ Collab.), J. of Physics: Conf. Series 632, 012089 (2015).

[26] Bartoli B., Bernardini P., Bi X., et al (ARGO-YBJ Collab.), Chinese Physics C 38, 4, 045001 (2014).

[27] Antoni T., Apel W. D., Badea A. F., et al., (KASCADE Collab.) Astroparticle Physics 24, p. 1-25 (2005).

[28] Apel W. D., Arteaga-Velazquez J. C., Bekk K., et al., (KASCADE-Grande Collab.) Astroparticle Physics 47, p. 54-66 (2013).

[29] Prosin V. V., Berezhnev S. F., Budnev N. M., et al., (Tunka Collab.) Nuclear Instruments and Methods in Physics Research A 756, p. 94-101 (2014).

[30] Rawlins K., Feusels T., et al., (IceCube Collab.) Proceedings of the 34th International Cosmic 
Ray Conference, (The Hague, The Netherlands 2015) PoS(ICRC2015) 334.

[31] Ivanov D., et al., (Telescope Array Collab.) Proceedings of the 34th International Cosmic Ray Conference, (The Hague, The Netherlands 2015) PoS(ICRC2015) 349.

[32] Berezhko E. G. Nucl. Phys. B (Proc. Suppl.) Vol. 256 - 257, 23-35 (2014); Berezhko E. G., Knurenko S. P., Ksenofontov L. T., Astropart. Phys. Vol. 36 31-36 (2012).

[33] Battistoni G., Ferrari A., Muraro S., et al., Proc. Suppl. Nucl. Phys. B. Vol. 168, 286 (2007).

[34] Dedenko L. G., Roganova T. M., Fedorova G. F., JETP Lett. Vol. 100, 223 (2014).

[35] Dedenko L. G., Roganova T. M., Fedorova G. F., Phys. Atom. Nucl. Vol. 78, N10, 894-902 (2015).

[36] Dedenko L. G., Lukyashin A. V., Fedorova G. F., et al., EPJ Web of Conferences Vol. 99, 10003 (2015).

[37] Dedenko L.G., Zatsepin G.T., Calculations of some characteristics of air showers taking fluctuations into account; Proceedings of the 6th International Cosmic
Ray Conference, (Moscow, USSR) Vol. II; Extensive air showers and cascades process, 201-208 (1960).

[38] Lagutin A. A., Tyumentsev A. G., Yushkov A. V., J. Phys. G., Vol. 30, 573-596 hep-ph/0402070Journal (2004).

[39] Dedenko L. G., Lukyashin A. V., Roganova T. M. et al., Bull. Russ. Acad. Sci. Phys. 81: 496 (2017).

[40] Dedenko L. G., Lukyashin A. V., Roganova T. M. et al., EPJ Web of Conferences Vol. 14516003 (2017).

[41] Dedenko L. G., Lukyashin A. V., Fedorova G. F., et al., Journal of Physics: Conference Series Vol. 798 012045 (2017).

[42] Dedenko L. G., Lukyashin A. V., Fedorova G. F., et al., EPJ Web of Conferences Vol. 158, 06006 (2017).

[43] Dedenko L. G., Lukyashin A. V., Fedorova G. F., et al., Journal of Physics: Conference Series Vol. 934, 012017 (2017).

[44] Glushkov A. V., Egorova V. P., Ivanov A. A., et al., Proceedings of the 28th International Cosmic Ray Conference, (Tsukuba, Japan) Vol. 1, 393 (2003). 